\title{
HSE Risk Assessment of Major Sewage Transport Tunnel Projects at the Construction Stage Based on the Structural Entropy Weight Method and the Cloud Model
}

\author{
Han Wu $\mathbb{D}^{1},{ }^{1}$ Sen Liu $\mathbb{D},{ }^{1}$ Denghui Liu $\mathbb{D}^{2},{ }^{2}$ and Junwu Wang $\mathbb{D}^{1}$ \\ ${ }^{1}$ School of Civil Engineering and Architecture, Wuhan University of Technology, Wuhan 430070, China \\ ${ }^{2}$ China Construction First Group Corporation Limited, Beijing 100161, China \\ Correspondence should be addressed to Junwu Wang; junwuwang@163.com
}

Received 15 June 2020; Revised 6 September 2020; Accepted 22 September 2020; Published 23 October 2020

Academic Editor: Haiyun Shi

Copyright (C) 2020 Han Wu et al. This is an open access article distributed under the Creative Commons Attribution License, which permits unrestricted use, distribution, and reproduction in any medium, provided the original work is properly cited.

\begin{abstract}
The health, safety, and environment (HSE) risk assessment of major sewage transport tunnel projects (MSTTPs) is of great significance to guarantee sewage treatment, ecological environment protection, and sustainable development. To accurately evaluate the HSE risk of MSTTPs at the construction stage and effectively deal with their randomness and ambiguity, a risk assessment model based on the structural entropy weight method (SEWM) and the cloud model is put forward in this paper. First, an index system for MSTTPs was constructed via a literature review and expert interviews, and the rough sets method was used to filter the indicators. Then, weights were calculated by the SEWM, which is able to consider both subjective and objective factors of the weight calculation. Finally, to clarify the randomness and ambiguity in the evaluation, the HSE risk level was determined by the cloud similarity. The model was applied to the Donghu Deep Tunnel Project in Wuhan, China, and the results demonstrated that its HSE risk level was medium, which was acceptable. The index related to construction safety had the largest weight. A humid environment, improper power utilization, and sludge and mud pollution were found to be the most influential risk indicators. The risk level could be intuitively and qualitatively judged by the figure evaluation cloud, providing a vivid and rapid evaluation tool for the emergency decision-making of project managers, and the risk level could be quantitatively judged by the calculation of cloud similarity. Moreover, through the comparison with gray correlation degree, set pair analysis, and fuzzy comprehensive evaluation method evaluation results, we prove the scientificity and effectiveness of the proposed model. The research results provide a valuable reference for the project management of MSTTPs at the construction stage.
\end{abstract}

\section{Introduction}

In the past 30 years, urbanization in developing countries, especially China, has progressed rapidly, resulting in increased difficulties in urban sewage treatment and environmental pollution problems [1]. Moreover, the capacity for sustainable development has been severely restricted. To solve these problems, major cities in developing countries are initiatively building major sewage transport tunnels under the core areas of the cities [2]. At present, a large number of major sewage transport tunnel projects (MSTTPs) are being built in China, and the total investment has exceeded 15 billion RMB [3].

MSTTPs are usually located 30-60 m underground and their construction sites are typically closed construction environments, which have great impacts on occupational safety. The construction processes of MSTTPs are characterized by complicated technology, a strict construction period, high mechanization, and complex construction safety risk [4]. The accidents and pollution incidents caused by these risk factors may result in huge economic losses and casualties, making the MSTTPs unable to be completed on time.

Health, safety, and the environment (HSE) is a concept that was originally generated in the petrochemical industry [5]. In recent decades, HSE has been gradually applied to high-risk industries [5] and the management of major projects [6] with established results. In combination with the research purposes of this study, the HSE risk of MSTTPs is 
defined as the occupational health risk of workers, the construction safety risk, and the environmental pollution risk caused by the construction of MSTTPs. The occupational health risk of workers (the $\mathrm{H}$ risk factor) is geared toward the research category of public health science [7]. Safety risk management during construction, which is related to the $S$ risk factor, belongs to the research areas of civil engineering and management science. The adverse effects of construction operations on the surrounding environment, which are related to the E risk factor, are part of the research fields of environmental engineering and civil engineering.

Referring to the general risk assessment process, the HSE risk assessment of MSTTPs in the present study includes three components, namely, the determination of risk indexes, weight calculations, and the selection of assessment methods.

At present, the existing research on HSE has primarily considered the HSE performance management of enterprises and the HSE risk management system of projects. Based on three elements, namely, time, scope, and type, Amir-Heidari et al. [8] comprehensively classified the HSE performance evaluation methods of enterprises and put forward a new HSE performance evaluation method from both positive and negative aspects. Lu and Li [9] studied the importance of the teamwork culture of construction teams in the HSE risk management system. Via a case analysis of a large prefabrication plant, it was proven that ensuring the HSE risk management system in an immersed tube prefabrication plant is of great significance to its smooth implementation. A team's strength could be mobilized and all construction workers could be motivated to participate in vocational education and safety production training. Zhang and $\mathrm{Li}[10]$ systematically studied the construction risk of the subsea tunnel of the Hong Kong-Zhuhai-Macao Bridge via HSE risk management. The LEC, an operation condition risk assessment, was used as the evaluation method for the analysis of the HSE risk level of the subsea tunnel, and the corresponding risk response measures were stated. However, the quantitative analysis and calculation process of the LEC method are subjective and do not provide a clear logical relationship between the values of risk indexes and the risk response measures. In addition, to the best of the authors' knowledge, a study on the HSE risk assessment of MSTTPs has not yet been reported. The main reason for the lack of prior research might be that the MSTTPs have only been restarted in the past few years, and many completed MSTTPs, such as the famous Thames Tunnel in London, were completed over a century ago.

The structural entropy weight method (SEWM) is a combined subjective and objective weight method that has been extensively used in recent years. Based on the characteristics of a large number of risk indicators for coalbed methane development and their complex relationships, Wang et al. [11] used the SEWM to process questionnaire survey results for weight calculation. The results were consistent with the actual situation of the case project. By employing the SEWM to calculate the weights of indexes, Liang et al. [12] not only effectively solved the problem of the lack of statistical data in the risk assessment of pipeline damage to the goaf, but also better handled the limitations of expert experience. Sun et al. [13] effectively adopted the SEWM to calculate the weights of the bearing capacity evaluation indexes of water resources.

For the selection of a risk assessment method, as one of the key steps in risk assessment, the characteristics of risk factors and assessment should be fully considered. Rezaee et al. [14] pointed out that the uncertainty of risk factors and the objectivity of assessment results are the key issues in the field of HSE risk assessment in the chemical industry. The failure mode and effects analysis (FMEA) and the fuzzy data envelopment analysis (DEA) were used to deal with these problems, but the randomness and fuzziness of the risk factors and risk assessment were neither further explored nor analyzed. The grey relational degree [15], set pair analysis [16], and fuzzy comprehensive evaluation [17] have been frequently used in risk assessment; while they have achieved sound results, it remains difficult for them to accurately describe randomness and fuzziness.

The cloud model is able to analyze both qualitative descriptions and quantitative data and can handle both fuzziness and randomness of a risk system. In recent years, it has become increasingly more widely used in the field of risk assessment. Liu et al. [18] combined the cloud model with an artificial neural network and proposed a new method of urban flood risk assessment that could effectively deal with the randomness and fuzziness of urban flood risk factors. Peng [19] used the positive cloud generator and the reverse cloud generator to construct a risk assessment model of a cable-stayed bridge; the risk assessment results based on the cloud model were found to be more stable and reasonable. Liu et al. [20] employed the cloud model to improve the traditional FMEA method, and their case study results proved the effectiveness of this amendment.

The present paper proposes a risk assessment model of MSTTPs based on the SEWM and the cloud model. The main contributions of this paper are as follows. (1) For the first time, a HSE risk evaluation index system was constructed from three aspects of health, safety, and environment by using the methods of literature research and questionnaire survey. Based on the genetic reduction algorithm of rough set theory, this paper retained the core evaluation index, effectively reduced the workload of the evaluation process, and enhanced the practicability of this method. (2) In this paper, the structural entropy weight method with the advantages of strong explanatory power and high accuracy of calculation results was used to calculate the weights of indexes. (3) Considering the fuzziness and randomness in the evaluation process, the qualitative concept of HSE risk evaluation index and the conversion of quantitative data were realized by the cloud model, which made the evaluation more reasonable. In addition, the risk level could be intuitively and qualitatively judged by the figure evaluation cloud, providing a vivid and rapid evaluation tool for the emergency decision-making of project managers, and the risk level could be quantitatively judged by the calculation of cloud similarity. (4) This paper revealed for the first time that, among the health, safety, and 
environment, the index related to safety had the largest weight, while the index affecting the environment had the smallest weight. This weight distribution was the same as the current research situation; the main research focused on the construction safety risks, while the research on analysis of construction's environmental impact risks was less. A humid environment, improper power utilization, and sludge and mud pollution were found to be the most influential risk indicators.

The remainder of this paper is organized as follows. Section 2 focuses on the research materials and methods, including the index system and the risk assessment method based on the SEWM and the cloud model. The process of the case analysis is presented in Section 3, Section 4 details the discussion of the results, and the conclusions are presented in Section 5.

\section{Materials and Methods}

\subsection{The Index System of HSE Risk Assessment of Major Sewage Transport Tunnel Projects}

2.1.1. Preliminary Selection of HSE Risk Index Indicators of MSTTPs. According to the principles of scientificity, systematicness, effectiveness, and operability, risk indicators of the categories of health, safety, and the environment were preliminarily identified via research of the existing literature. Table 1 presents references for each risk indicator.

2.1.2. Determination of HSE Risk Index Indicators of MSTTPs. At present, there only exist a handful of MSTTPs that have been constructed around the world. As many were built long ago, they are of little significance for investigation. In addition, China and other countries have just begun to construct MSTTPs. Therefore, the available engineering data of MSTTPs is scarce. Considering this, the expert interview method was chosen to score the importance of the 42 HSE risk indicators that have been identified. According to the index score, the rough set was used to filter the index with the help of Rosetta.

In the questionnaire developed for this research, experts scored each indicator on a 5-point Likert scale. Each secondary indicator has five degrees of attributes for its primary indicator, which are "very strong" (5 points), "strong" (4 points), "average" (3 points), "weak" ( 2 points), and "very weak" (1 point).

The questionnaire was distributed to twenty-five experts with rich theoretical and practical experience. Eight experts were affiliated with universities, eleven were affiliated with construction enterprises of MSTTPs, and five were affiliated with MSTTP design units. Seventeen experts had the title of senior engineer or associate professor, while the other eight experts had the title of deputy senior engineer. Twenty experts had participated in the Donghu Deep Tunnel Project in Wuhan, which is the case study object of this article. Eleven experts had participated in more than three MSTTPs. These characteristics of the experts ensure the rationality of the survey results.
TABLE 1: Identification results of HSE risk indicators.

\begin{tabular}{|c|c|c|}
\hline Criteria layer & Preliminary indicator & References \\
\hline \multirow{8}{*}{ Health } & Construction noise & [21] \\
\hline & Construction dust & {$[22]$} \\
\hline & Humid environment & {$[23]$} \\
\hline & High ambient temperature & {$[23]$} \\
\hline & Irregular lifestyle & {$[24]$} \\
\hline & Toxic and harmful chemicals & {$[7]$} \\
\hline & Whole body vibration & {$[7]$} \\
\hline & Radiation & {$[25]$} \\
\hline \multirow{26}{*}{ Safety } & Unreasonable tunneling parameters & {$[26]$} \\
\hline & Excessive deformation & {$[26]$} \\
\hline & Improper use of transportation & {$[2]$} \\
\hline & Wear failure of the shield cutter head & {$[27]$} \\
\hline & Improper power utilization & {$[28]$} \\
\hline & Unqualified shield segments & {$[29]$} \\
\hline & $\begin{array}{l}\text { Improper operation of shield } \\
\text { segments }\end{array}$ & {$[30]$} \\
\hline & Random stacking & {$[2]$} \\
\hline & Incorrect protection & {$[31]$} \\
\hline & Failure of bearing seal & {$[32]$} \\
\hline & Failure of hoisting jack & {$[32]$} \\
\hline & Clogging at the exit of slurry & {$[32]$} \\
\hline & Bad grouting effect & [32] \\
\hline & Improper selection of TBM machine & {$[33]$} \\
\hline & Inadequate fastening of bolt & {$[34]$} \\
\hline & Anchor bolt quality & [34] \\
\hline & $\begin{array}{l}\text { Imprecise installation of launching } \\
\text { base }\end{array}$ & {$[34]$} \\
\hline & Deviation of TBM from target shaft & {$[34]$} \\
\hline & $\begin{array}{c}\text { Formation of "mud cake" during } \\
\text { excavation }\end{array}$ & [34] \\
\hline & Derailment or collision of electric cart & {$[34]$} \\
\hline & Grout pipe blockage & {$[34]$} \\
\hline & Improper grouting amount & {$[34]$} \\
\hline & $\begin{array}{c}\text { Improper control of grouting } \\
\text { coefficient }\end{array}$ & [34] \\
\hline & Leakage of pipe piece joints & {$[35]$} \\
\hline & Shield tail seal leakage & {$[35]$} \\
\hline & Segment seal failure & {$[36]$} \\
\hline \multirow{8}{*}{ Environment } & Domestic waste pollution & {$[37]$} \\
\hline & Exhaust pollution & {$[38]$} \\
\hline & Wastewater pollution & {$[37]$} \\
\hline & Sludges and muds pollution & [39] \\
\hline & Pollution from vehicles & {$[40]$} \\
\hline & Impact on groundwater level & {$[41]$} \\
\hline & Excessive surface subsidence & {$[42]$} \\
\hline & Destruction of the ecological structure & {$[43]$} \\
\hline
\end{tabular}

After twenty-five experts had completed scoring for all 42 secondary indicators, the results of the questionnaire were collected.

Rough sets theory is a mathematical method proposed by the Polish scientist Z. Pawlak to deal with inaccurate, uncertain, and incomplete data [44]. In the process of rough set reduction, the relationship between evaluation indicators and decision indicators can be established according to methods such as self-information and mutual information, so as to determine the importance of conditional attributes and calculate weights [45]. The Rosetta system is a tabular logic data tool based on the rough set theoretical framework. 
It was developed by the Department of Computer and Information Science of the Norwegian University of Science and Technology and the Institute of Mathematics, University of Warsaw, Poland. This software is not aimed at a specific research field, but a general tool for simplifying models.

In this paper, rough sets theory was used to screen indicators with the help of Rosetta system; the index screening results are shown in Table 2.

2.1.3. Construction of HSE Risk Index System of MSTTPs. According to the identified indicators of the HSE risk of MSTTPs, an index system including 3 primary indicators and 13 secondary indicators was constructed, as shown in Table 2. In addition, based on the characteristics and actual situations of MSTTPs, the connotation of each secondary indicator was analyzed, as presented in Table 2.

\subsubsection{HSE Risk Assessment Criteria of MSTTPs.} Currently, no uniform HSE risk evaluation standard does exist [46]. In this paper, the HSE risk was divided into five levels, namely, lower risk, low risk, medium risk, high risk, and higher risk. The scoring interval was set as $[0,100]$. According to the opinions of experts, the risk levels were divided and are presented in Table 3. The concepts and calculations of Ex, En, and $\mathrm{He}$ are described in the subsequent section.

Lower risk indicates that the risk is negligible. Low risk indicates that the risk is low, but project managers must check the existing risk management measures. Medium risk indicates that the risk is moderate and acceptable, but further measures should be considered. High risk indicates that the risk is serious and mitigation measures should be taken immediately. Finally, higher risk indicates that the risk is the most serious and completely unacceptable and construction should be stopped immediately.

\subsection{The Assessment Model of HSE Risk Assessment of Major Sewage Transport Tunnel Projects}

2.2.1. Introduction of the SEWM. The SEWM combines both qualitative analysis and the quantitative analysis of weight calculation [11]. Its basic logic is as follows. First, the Delphi expert survey method and fuzzy analysis method are combined to form a quantitative order that fully considers the subjective judgments of experts. Then, entropy computing and cognitive blind degree analysis are used to analyze the quantitative order, and the statistical processing of potential data that may produce deviation is conducted with the modified uncertainty of the subjective judgments of experts. Finally, the weights of indexes are obtained via normalization.

2.2.2. Introduction of the Cloud Model. The cloud model was first proposed by Li et al., a Chinese scholar. It realizes the two-way transformation of qualitative concepts and quantitative values [47]. By applying the cloud model to the evaluation of complex systems, the randomness and fuzziness of indicators and evaluation are fully considered [18].
A qualitative concept can be described by Ex (expected value), En (entropy), and He (hyper entropy), which are also recorded as (Ex, En, and He) [19].

(1) Ex represents the basic certainty of the qualitative concept. It is the mathematical expectation of the cloud drop in the spatial distribution of the domain. It also denotes the center value of the evaluation index in a certain grade score interval.

(2) En represents the uncertainty measure of the qualitative concept and is determined by both randomness and ambiguity of the concept. It evaluates the degree of fuzziness of the index grade boundary.

(3) $\mathrm{He}$ is the uncertainty of entropy and reflects the degree of deviation of the random variable corresponding to the qualitative concept from the normal distribution.

2.2.3. Risk Assessment Method of MSTTPs Based on the SEWM and the Cloud Model. The flow chart of this evaluation method is illustrated in Figure 1.

The specific steps of the risk assessment method of MSTTPs based on the SEWM and the cloud model are as follows.

Step 1. Determine the standard cloud of each risk level.

There are 5 subintervals shown in Table 1. Let the subinterval $i$ be $\left[\mathrm{C}_{i}^{\min }, \mathrm{C}_{i}^{\max }\right]$, and the digital characteristic values are

$$
\left\{\begin{array}{l}
\mathrm{Ex}_{i}=\frac{\left(C_{i}^{\max }+C_{i}^{\min }\right)}{2}, \\
\mathrm{En}_{i}=\frac{\left(C_{i}^{\max }-C_{i}^{\min }\right)}{(2 \sqrt{2 \operatorname{In} 2})}, \\
\mathrm{He}_{i}=k,
\end{array}\right.
$$

where $k$ is a constant that is usually assigned the value of 0.5 [47].

Step 2. Calculate the weights by the SEWM.

The detailed steps for determining the weights are as follows [13].

(1) Determine the Quantitative Order Based on Expert Opinions. According to the operating procedures of the Delphi method, $k$ experts with rich theoretical and practical experience are selected and sent a questionnaire. The questionnaire must be completed anonymously.

Experts score the importance of each index by using the integer of $(1-n)$, where 1 is the most important index and $n$ is the least important index. The number of indicators is $n$. The index ranking matrix $\mathbf{A}$ is obtained as follows: 
TABLE 2: The index system for the HSE risk assessment of MSTTPs.

\begin{tabular}{|c|c|c|}
\hline Criteria layer & Indicator layer & Indicator interpretation \\
\hline \multirow{4}{*}{ Health } & Construction noise $\mathrm{H} 1$ & $\begin{array}{l}\text { Shield machines and vehicles in tunnels generate noise, which can give hearing damage to } \\
\text { construction workers. }\end{array}$ \\
\hline & Humid environment $\mathrm{H} 2$ & $\begin{array}{c}\text { The humidity in the tunnel is exceedingly high, which may lead to skin dipping and } \\
\text { erosion of construction workers. }\end{array}$ \\
\hline & High ambient temperature $\mathrm{H} 3$ & Excessive ambient temperature may cause heatstroke in workers. \\
\hline & $\begin{array}{l}\text { Toxic and harmful chemicals } \\
\text { H4 }\end{array}$ & $\begin{array}{l}\text { Cement, paint, and other items may cause workers to suffer from occupational diseases } \\
\text { such as contact dermatitis. }\end{array}$ \\
\hline \multirow{4}{*}{ Safety } & Excessive deformation S1 & $\begin{array}{c}\text { Excessive deformation leads to the destruction of the original structure, leading to safety } \\
\text { accidents. }\end{array}$ \\
\hline & Improper power utilization S2 & $\begin{array}{l}\text { If the temporary power management is improper, it may easily give rise to an electric } \\
\text { shock accident. }\end{array}$ \\
\hline & $\begin{array}{l}\text { Improper operation of shield } \\
\text { segments } \mathrm{S} 3\end{array}$ & $\begin{array}{l}\text { The improper operation of the construction personnel would end up in segments falling, } \\
\text { causing object strike accidents. }\end{array}$ \\
\hline & $\begin{array}{l}\text { Imprecise installation of } \\
\text { launching base S4 }\end{array}$ & $\begin{array}{l}\text { The launch base was installed incorrectly and the shield machine could not launch } \\
\text { normally, resulting in a safety accident. }\end{array}$ \\
\hline \multirow{5}{*}{ Environment } & Domestic waste pollution E1 & $\begin{array}{c}\text { Domestic waste in office and living areas may cause damage to the surrounding } \\
\text { environment. }\end{array}$ \\
\hline & Exhaust pollution E2 & $\begin{array}{l}\text { The construction of tunnels uses a lot of machinery, and waste gas will be generated } \\
\text { during use. If it is not properly treated, it will pollute the atmosphere. }\end{array}$ \\
\hline & Sludges and muds pollution E3 & $\begin{array}{c}\text { Due to inexperience, it is difficult to achieve zero discharge of construction waste such as } \\
\text { engineering sludges and muds. }\end{array}$ \\
\hline & Impact on groundwater E4 & $\begin{array}{c}\text { The earth pressure is not set properly, which may compact the surrounding soil and cause } \\
\text { the groundwater level to rise significantly. }\end{array}$ \\
\hline & Excessive surface subsidence E5 & $\begin{array}{c}\text { During the construction of the shield, the surrounding soil is disturbed greatly, and the } \\
\text { ground surface has a large settlement. }\end{array}$ \\
\hline
\end{tabular}

Table 3: Standard risk level classification and corresponding cloud models.

\begin{tabular}{lcccc}
\hline Risk level & Standards & Ex & En & He \\
\hline Lower risk & {$[0,25]$} & 12.5 & 10.616 & 0.5 \\
Low risk & $(25,50]$ & 37.5 & 10.616 & 10.616 \\
Medium risk & $(50,75]$ & 62.5 & 6.5 & 0.5 \\
High risk & $(75,90]$ & 82.5 & 4.246 & 0.5 \\
Higher risk & $(90,100]$ & 95.0 & 0.5 \\
\hline
\end{tabular}

$$
\mathbf{A}=\left[\begin{array}{ccc}
a_{11} & \cdots & a_{1 n} \\
\vdots & \ddots & \vdots \\
a_{k 1} & \cdots & a_{k n}
\end{array}\right]
$$

where $a_{i j}$ indicates the importance evaluation of expert $i$ $(1 \leq i \leq k)$ for indicator $j(1 \leq j \leq n)$.

(2) Perform Weight Correction on the Quantitative Order. According to the concept of the entropy weight method, the uncertainty of the quantitative order is corrected [11]:

$$
\mu(I)=-\frac{\operatorname{In}(m-I)}{\operatorname{In}(m-1)},
$$

where $I$ is the qualitative ranking number given by experts. If the ranking number given is 1 , then $I$ is 1 . If the ranking number is 2 , then $I$ is 2 . Additionally, $m$ is the conversion parameter, which is generally considered as $m=j+2$.

After including $a_{i j}$ in (3), the ordering quantitative conversion value $b_{i j}$ is obtained. Assuming that all experts have the same weights in the evaluation system, the average cognitive degree is defined as [12]

$$
b_{i j}=\frac{\left(b_{1 j}+b_{2 j}+\cdots+b_{k j}\right)}{k} .
$$

The uncertainty of experts' cognition is defined as knowledge blindness, and its parameter $Q_{j}$ is as given by the following equation [12]:

$$
Q_{j}=\left|\frac{\left\{\left[\max \left\{b_{1 j}, b_{2 j}, \ldots, b_{k j}\right\}-b_{j}\right]+\left[\min \left\{b_{1 j}, b_{2 j}, \ldots, b_{k j}\right\}-b_{j}\right]\right\}}{2}\right| .
$$

The overall cognition degree of indicator $j$ by $k$ experts is defined as [13]

$$
x_{j}=b_{j}\left(1-Q_{j}\right) .
$$




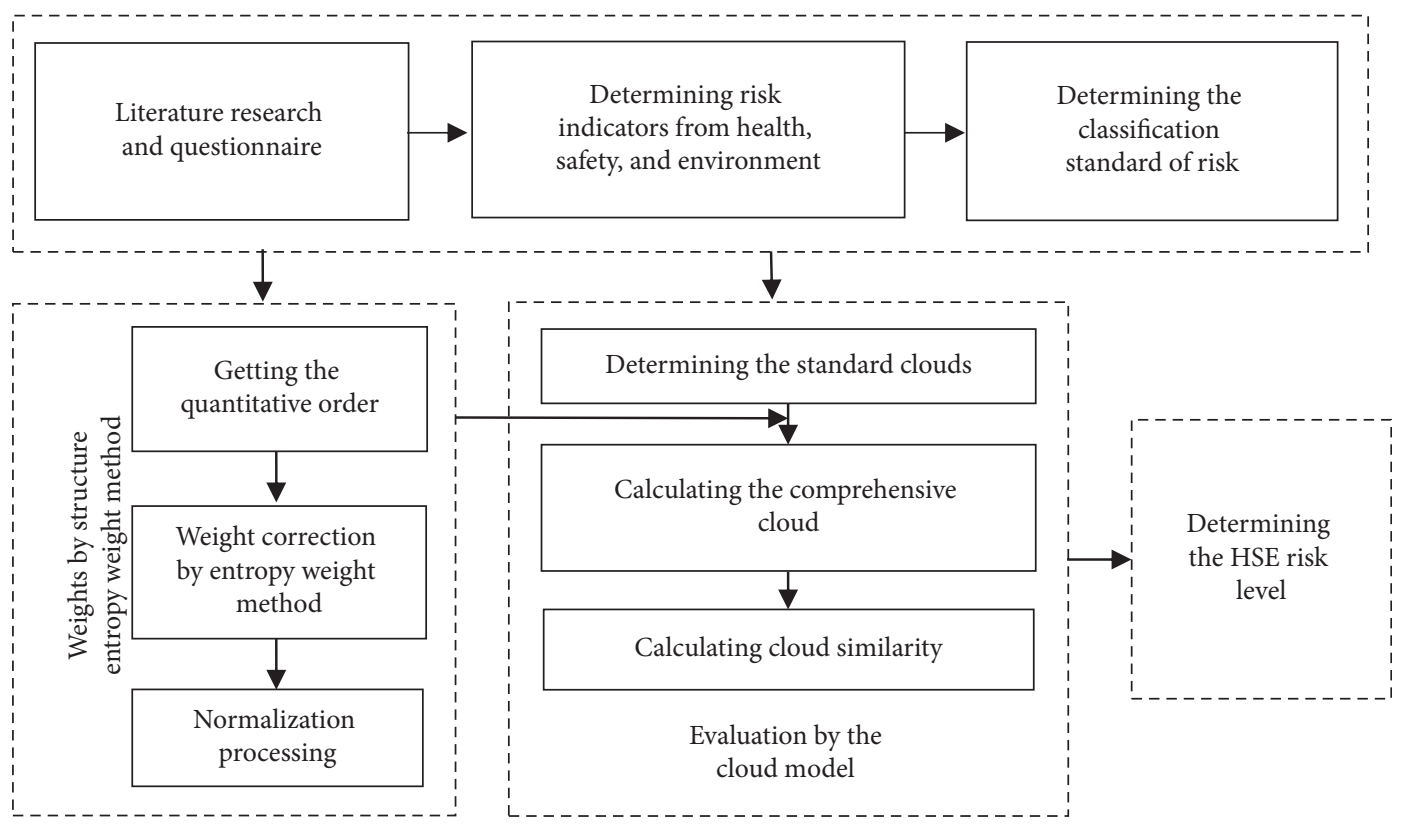

FIGURE 1: Flow chart of risk assessment method of MSTTPs based on the SEWM and the cloud model.

(3) Perform Normalization to Determine the Weights.

$$
\omega_{j}=\frac{x_{j}}{\sum_{j=1}^{n} x_{j}} .
$$

Step 3. Calculate the clouds of the evaluation factors and the comprehensive cloud.

By substituting the scores of all experts $\left[z_{i j}\right]_{k \times m}$ into the following equations, the clouds of the evaluation factors are obtained [19]:

$$
\begin{aligned}
\mathrm{Ex}_{j} & =\frac{1}{k} \sum_{i=1}^{k} z_{i j}, \\
\mathrm{En}_{j} & =\sqrt{\frac{\pi}{2}} \times \frac{1}{k} \sum_{i=1}^{k}\left|z_{i j}-\mathrm{Ex}_{j}\right|, \\
\mathrm{He}_{j} & =\sqrt{\left|S_{j}^{2}-\mathrm{En}_{j}^{2}\right|} .
\end{aligned}
$$

The weights $\mathbf{W}=\left(\omega_{1}, \omega_{2}, \ldots, \omega_{n}\right)^{\mathrm{T}}$ obtained in Step 2 are substituted into the clouds of the evaluation factors $\left(\mathrm{Ex}_{j}, \mathrm{En}_{j}, \mathrm{He}_{j}\right)$. The comprehensive cloud of the HSE risk of the MSTTP is determined by the following equation [19]:

$$
\left\{\begin{array}{l}
\mathrm{Ex}=\sum_{j=1}^{n} \mathrm{Ex}_{j} \cdot \omega_{j}, \\
\mathrm{En}=\sqrt{\sum_{j=1}^{n} \mathrm{En}_{j}^{2} \cdot \omega_{j}}, \mathrm{He}=\sum_{j=1}^{n} \mathrm{He}_{j} \cdot \omega_{j} .
\end{array}\right.
$$

By drawing the comprehensive cloud and five standard clouds in the same image, the HSE risk level of an MSTTP can be qualitatively judged.
Step 4. Determine the HSE risk level by the cloud similarity.

The steps for the calculation of $\delta_{i}$, which is the cloud similarity between the clouds of the evaluation factors and the comprehensive cloud, are as follows [47]:

(1) Generate $\operatorname{Ex}_{p}=\operatorname{Norm}\left(E n, \mathrm{He}^{2}\right)$.

(2) Generate $x_{p}=\operatorname{Norm}\left(\mathrm{Ex}, \mathrm{Ex}_{p}^{2}\right)$.

(3) Substitute into (12) to calculate $\mu_{p}$ :

$$
\mu_{p}=e^{-\left(\left(x_{p}-E x_{i}^{2}\right)^{1} /\left(2 \mathrm{En}_{i}^{2}\right)\right)} .
$$

(4) Repeat Steps 2 and 3 to generate $\mu_{p}$, where $q \in[10,30]$. Then, $\delta_{i}$ can be calculated as follows:

$$
\delta_{i}=\frac{1}{q} \sum_{p=1}^{q} \mu_{p} .
$$

The greater the value of $\delta_{i}$ is, the closer the HSE risk level of the MSTTP is to the evaluation level. In this way, the HSE risk level of an MSTTP can be calculated quantitatively.

\section{Results}

3.1. Engineering Background. The case selected in this article is the Donghu Deep Tunnel Project in Wuhan, which is the largest MSTTP in China. Its recent service scope covers the core area of Wuhan, which is about $130.35 \mathrm{~km}^{2}$, and its long-term control service range is about $200.25 \mathrm{~km}^{2}$. Its total length is about $17.5 \mathrm{~km}$ via shield construction, the tunnel diameters are D3000-D3400 mm, and the buried depths are $30-50 \mathrm{~m}$. When this project is successfully completed, the sewage from the core area of Wuhan will be directly transferred to the Beihu Sewage Treatment Plant, which is the largest sewage treatment plant in 
China. The Donghu Deep Tunnel not only can elevate the capacity and efficiency of urban sewage transport, but can also effectively avoid the problem of the mixture of rain and sewage, thereby improving the water environment of Shahu Port, Luojia Port, and Donghu Port. To achieve the smooth implementation of this project, it is necessary to carry out a typical HSE risk assessment.

Current data of MSTTPs was difficult to obtain, so the data used for the computations of the weights and risk levels in the case study were obtained by questionnaires. The 10 invited experts were the experts who selected the HSE risk indicator system in the preliminary study. The 10 experts scored the indexes on the basis of fully understanding the site situation, construction content, and process of the Donghu Deep Tunnel Project. According to (1), three digital clouds of the characteristics of each level were computed and are presented in Table 1.

3.2. Determination of Indicator Weights. After a questionnaire survey, the results of the importance scores of the 3 primary indicators and 15 secondary indicators by the twenty-five experts were substituted into (2)-(7), and the calculation results of the weights of the 3 primary indexes (Table 4), 4 secondary indexes related to the health indicator (Table 5), 4 secondary indexes related to the safety indicator (Table 6), and 5 secondary indexes related to the environment indicator (Table 7) were obtained.

From Table 4, it can be seen that the safety indicator has the largest weight of the primary indexes, while the environment indicator has the smallest weight. This weighting explains the phenomenon of most previous research focusing on construction safety risks instead of environmental risks.

Table 5 shows that the humid environment index $(\mathrm{H} 2)$ has the largest weight, while the high ambient temperature $(\mathrm{H} 3)$ has the least weight. These results are consistent with the construction content of MSTTPs, in which earth pressure balance shield machines are used for shield construction and typically create a high-humidity environment. However, MSTTPs are mostly constructed underground and with good ventilation. So, high temperature conditions will not occur.

As presented in Table 6, the weight of the improper power utilization index (S2) is the largest of the 6 secondary indexes related to safety, and the weight of the imprecise installation of launching base (S4) is the smallest. The construction safety risk management of MSTTPs should therefore focus on improper power utilization.

As presented in Table 7, the sludge and mud polarization index (E3) has the largest weight. The reason for this is that the main pollutants produced during the shield construction of the Donghu Deep Tunnel Project in Wuhan are sludge and mud.

3.3. Calculations of the Clouds of Evaluation Factors and the Comprehensive Cloud. Via the questionnaire survey results of 25 experts, the scores of the 13 secondary indexes of the Donghu Deep Tunnel Project were averaged and introduced into (8)-(10). The evaluation cloud model of each index was obtained, as presented in Table 8 .
TABLE 4: The weights of primary indexes.

\begin{tabular}{lccc}
\hline No. & $\mathrm{H}$ & $\mathrm{S}$ & $\mathrm{E}$ \\
\hline Expert 1 & 2 & 1 & 3 \\
Expert 2 & 1 & 2 & 3 \\
Expert 3 & 2 & 1 & 3 \\
Expert 4 & 3 & 1 & 2 \\
Expert 5 & 2 & 1 & 3 \\
Expert 6 & 2 & 1 & 3 \\
$\ldots$ & $\ldots$ & $\ldots$ & $\ldots$ \\
Expert 24 & 2 & 1 & 3 \\
Expert 25 & 1 & 0.9253 & 3 \\
$b_{j}$ & 0.7687 & 0.0291 & 0.5985 \\
$Q_{j}$ & 0.0187 & 0.8984 & 0.1515 \\
$x_{j}$ & 0.7543 & 0.4158 & 0.5078 \\
$\omega_{j}$ & 0.3491 & 1 & 0.2350 \\
Ranking & 2 & & 3 \\
\hline
\end{tabular}

TABLE 5: The weights of 4 secondary indexes related to health.

\begin{tabular}{lcccc}
\hline No. & $\mathrm{H} 1$ & $\mathrm{H} 2$ & $\mathrm{H} 3$ & $\mathrm{H} 4$ \\
\hline Expert 1 & 1 & 3 & 2 & 4 \\
Expert 2 & 2 & 1 & 3 & 4 \\
Expert 3 & 2 & 1 & 4 & 3 \\
Expert 4 & 3 & 1 & 4 & 2 \\
Expert 5 & 3 & 1 & 4 & 2 \\
Expert 6 & 2 & 3 & 4 & 1 \\
$\ldots$ & $\ldots$ & $\ldots$ & $\ldots$ & $\ldots$ \\
Expert 24 & 2 & 3 & 4 & 1 \\
Expert 25 & 3 & 2 & 1 & 4 \\
$b_{j}$ & 0.8101 & 0.9159 & 0.5929 & 0.6446 \\
$Q_{j}$ & 0.0948 & 0.0746 & 0.1224 & 0.0708 \\
$x_{j}$ & 0.7333 & 0.8476 & 0.5203 & 0.5990 \\
$\omega_{j}$ & 0.2716 & 0.3139 & 0.1927 & 0.2218 \\
Ranking & 2 & 1 & 4 & 3 \\
\hline
\end{tabular}

TABLE 6: The weights of 6 secondary indexes related to safety.

\begin{tabular}{lcccc}
\hline No. & S1 & S2 & S3 & S4 \\
\hline Expert 1 & 3 & 2 & 1 & 4 \\
Expert 2 & 4 & 1 & 2 & 3 \\
Expert 3 & 2 & 1 & 3 & 4 \\
Expert 4 & 4 & 2 & 1 & 3 \\
Expert 5 & 4 & 1 & 3 & 2 \\
Expert 6 & 4 & 1 & 2 & 3 \\
$\ldots$ & $\ldots$ & $\ldots$ & $\ldots$ & $\ldots$ \\
Expert 9 & 3 & 2 & 1 & 4 \\
Expert 10 & 3 & 1 & 2 & 4 \\
$b_{j}$ & 0.6485 & 0.9191 & 0.8274 & 0.5741 \\
$Q_{j}$ & 0.0668 & 0.0778 & 0.1120 & 0.1413 \\
$x_{j}$ & 0.6052 & 0.8476 & 0.7347 & 0.4930 \\
$\omega_{j}$ & 0.2258 & 0.3162 & 0.2741 & 0.1839 \\
Ranking & 3 & 1 & 2 & 4 \\
\hline
\end{tabular}

After substituting the weight calculation results and the clouds of the evaluation factors into (11), the comprehensive cloud was found to be $67.8774,7.3989$, and 1.4578 , and this is shown in Figure 2.

It can be easily and intuitively seen that the HSE risk level of the Donghu Deep Tunnel Project was found 
TABLE 7: The weights of 5 secondary indexes related to the environment.

\begin{tabular}{|c|c|c|c|c|c|}
\hline No. & E1 & E2 & E3 & E4 & E5 \\
\hline Expert 1 & 5 & 4 & 1 & 3 & 2 \\
\hline Expert 2 & 4 & 3 & 2 & 1 & 5 \\
\hline Expert 3 & 4 & 5 & 1 & 2 & 3 \\
\hline Expert 4 & 3 & 5 & 2 & 1 & 4 \\
\hline Expert 5 & 5 & 4 & 3 & 1 & 2 \\
\hline Expert 6 & 4 & 5 & 1 & 2 & 3 \\
\hline$\ldots$ & $\ldots$ & $\ldots$ & $\ldots$ & $\ldots$ & $\ldots$ \\
\hline Expert 24 & 4 & 5 & 2 & 1 & 3 \\
\hline Expert 25 & 5 & 4 & 1 & 3 & 2 \\
\hline$b_{j}$ & 0.5483 & 0.5621 & 0.9272 & 0.8453 & 0.7891 \\
\hline$Q_{j}$ & 0.0320 & 0.1314 & 0.0403 & 0.0388 & 0.0956 \\
\hline$x_{j}$ & 0.5308 & 0.4882 & 0.8898 & 0.8126 & 0.7136 \\
\hline$\omega_{j}$ & 0.1545 & 0.1421 & 0.2590 & 0.2366 & 0.2077 \\
\hline Ranking & 4 & 5 & 1 & 2 & 3 \\
\hline
\end{tabular}

TABle 8: The clouds of evaluation factors.

\begin{tabular}{lcccc}
\hline Indicator & Average score & Ex & En & $\mathrm{He}$ \\
\hline H1 & 78.80 & 78.80 & 7.798650 & 1.617801 \\
H2 & 84.84 & 84.84 & 5.120245 & 0.503215 \\
H3 & 56.56 & 56.56 & 8.103378 & 2.329037 \\
H4 & 73.96 & 73.96 & 8.556462 & 0.003187 \\
S1 & 70.48 & 70.48 & 6.639879 & 2.580660 \\
S2 & 55.48 & 55.48 & 7.008761 & 0.473030 \\
S3 & 71.24 & 71.24 & 6.864416 & 0.826566 \\
S4 & 79.44 & 79.44 & 6.287035 & 0.010070 \\
E1 & 66.80 & 66.80 & 9.021574 & 2.986067 \\
E2 & 61.84 & 61.84 & 7.483897 & 2.767690 \\
E3 & 52.28 & 52.28 & 9.657094 & 3.233065 \\
E4 & 54.48 & 54.48 & 6.371236 & 3.124658 \\
E5 & 56.60 & 56.60 & 9.342341 & 2.485330 \\
\hline
\end{tabular}

to be the closest to moderate risk $[18,38]$. The cloud model could therefore effectively deal with the randomness and ambiguity in the HSE risk evaluation of MSTTPs.

3.4. Determination of the HSE Risk Level by the Cloud Similarity. After introducing the obtained comprehensive evaluation cloud $(67.8774,7.3989$, and 1.4578$)$ into (12) and (13), the cloud similarities $\delta_{i}$ between the comprehensive evaluation cloud and the five standard clouds were obtained and are presented in Table 9. In (12), $q$ is an important parameter $(q \in[10,30]) . \delta_{i}$ under three different values of $q$ was calculated in Table 9.

The cloud similarity between the comprehensive evaluation cloud and the standard Cloud 3 was the largest, which indicates that the HSE risk level of the Donghu Deep Tunnel Project is medium risk.

\section{Discussion}

The weight calculation in risk assessment often only considers subjective or objective factors, thereby making the weights inaccurate. Moreover, the common risk evaluation methods cannot reasonably take into account the randomness and ambiguity in the evaluation process, thereby making the risk evaluation results inaccurate. Currently, MSTTPs are under construction in large quantities, but there is little research on the risk of MSTTPs, and research on the HSE risk assessment of MSTTPs has not yet been reported.

To fill this gap, a typical risk evaluation index system of MSTTPs was constructed in this study for the first time. Then, to ensure the accuracy of the weight calculations, the SEWM, in which both subjective and objective factors in the weight calculation are comprehensively considered, was used to calculate the weights. Finally, an evaluation method based on the cloud model was adopted to describe the randomness and ambiguity of the HSE risk assessment of MSTTPs. The research results obtained in this study can help ensure the smooth implementation of MSTTPs and are of great significance for ensuring wastewater treatment, ecological environment protection, and sustainable development.

Because there are no existing research results on the HSE risk of MSTTPs, this paper compared the results of case analysis with the results of common risk assessment methods (the grey relational degree [15], set pair analysis [16], and fuzzy comprehensive evaluation [17]). The grey relational degree calculation results of each risk level are shown in Table 10. According to the calculation result of the index weight of Donghu Deep Tunnel Project and the average of the scores of each risk index, the gray correlation degree of Donghu Deep Tunnel Project was 0.428 , which belonged to medium risk. The calculation result of the five-element connection number of Donghu Deep Tunnel Projects calculated by set pair analysis is shown in Table 11 . When the confidence $\epsilon[0.50,0.70]$, the HSE risk of Donghu Deep Tunnel Project was medium risk. The evaluation results of fuzzy comprehensive evaluation are shown in Table 12. According to the principle of maximum membership degree of comprehensive evaluation, the HSE risk was medium risk.

The grey relational degree, set pair analysis, and fuzzy comprehensive evaluation results of HSE risk of Donghu Deep Tunnel Project belonged to the medium risk, and the direct evaluation results were basically consistent 


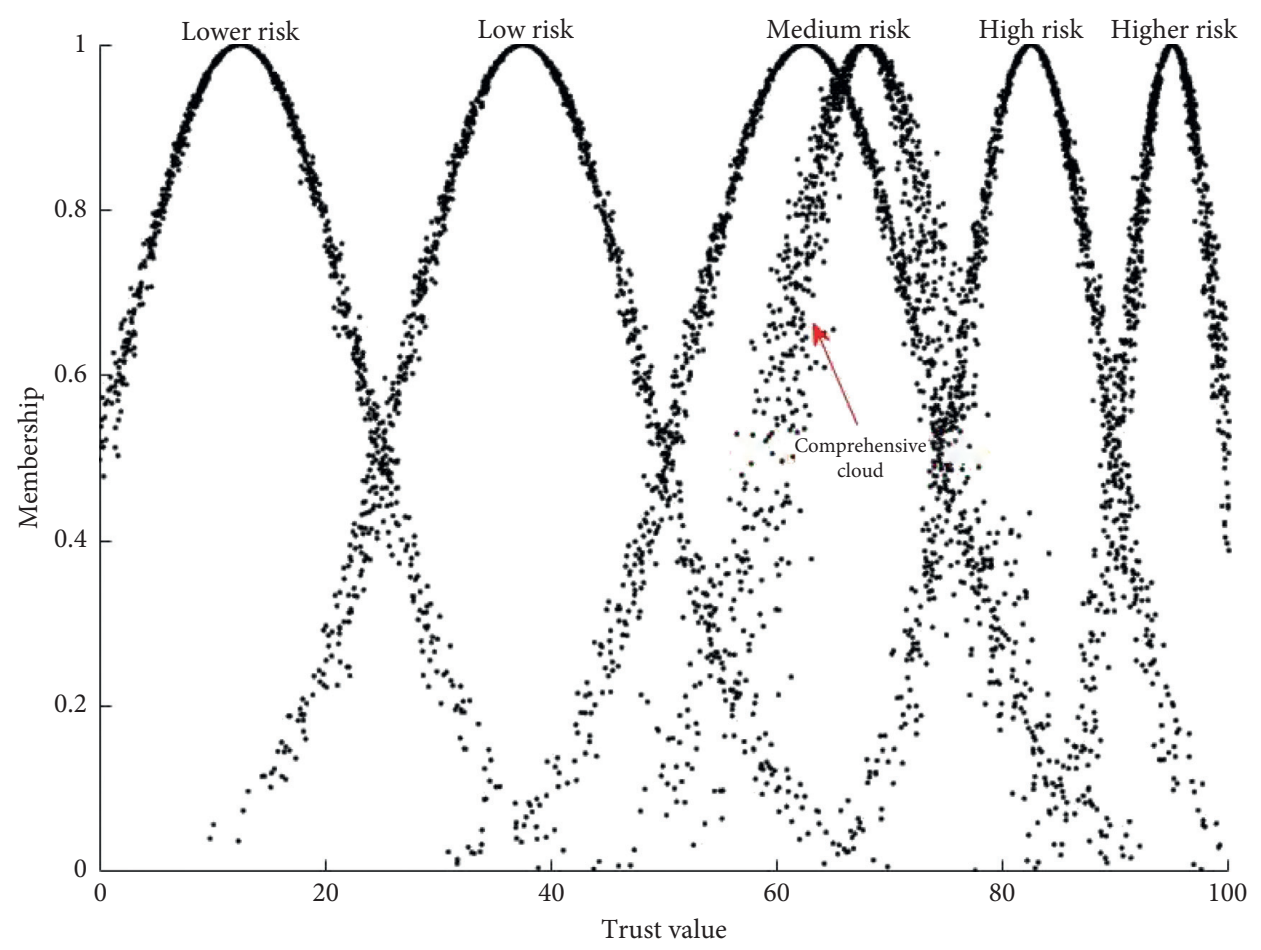

Figure 2: Cloud of comprehensive evaluation.

TABLE 9: Calculation results of the cloud similarity.

\begin{tabular}{lcccccc}
\hline Standard cloud & $q$ & Cloud 1 & Cloud 2 & Cloud 3 & Cloud 4 & Cloud 5 \\
\hline Cloud similarity $\delta_{i}$ & 10 & 0.003 & 0.121 & 0.614 & 0.262 & 0.010 \\
Cloud similarity $\delta_{i}$ & 20 & 0.002 & 0.120 & 0.620 & 0.251 & 0.007 \\
Cloud similarity $\delta_{i}$ & 30 & 0.000 & 0.125 & 0.615 & 0.254 \\
\hline
\end{tabular}

TABle 10: Comprehensive grey relational degree.

\begin{tabular}{lccccc}
\hline Risk level & Lower risk & Low risk & Medium risk & High risk & Higher risk \\
\hline Grey relational degree & 0.6667 & 0.5000 & 0.4000 & 0.3571 & 0.3333 \\
\hline
\end{tabular}

TABle 11: The results of Donghu Deep Tunnel Project' five-element contact numbers.

\begin{tabular}{lcccccc}
\hline Five-element contact numbers & $f_{1}$ & $f_{2}$ & $f_{3}$ & $f_{4}$ & $f_{5}$ & Level \\
\hline Donghu deep tunnel project & 0.0000 & 0.1080 & 0.5009 & 0.3569 & 0.0342 & Medium risk \\
\hline
\end{tabular}

Table 12: Evaluation results of the Donghu Deep Tunnel Project.

\begin{tabular}{lccccc}
\hline Risk level & Lower risk & Low risk & Medium risk & High risk & Higher risk \\
\hline Membership & 0.000 & 0.1107 & 0.5931 & 0.2787 & 0.0175 \\
\hline
\end{tabular}

with the evaluation results of the cloud model. The gray correlation model solved the ambiguity of indicators, but it was difficult for it to deal with the randomness and uncertainty of indicators $[15,47]$. When many pieces of data and information were used, the evaluation of indicators always carried a certain degree of uncertainty and randomness. In the application process of the set pair analysis method, it was difficult to determine the ambiguity of the index difference coefficient, so it was not able to be widely promoted in actual evaluation [16]. In practical applications, the fuzzy synthesis method often used the membership function to calculate the membership degree of each index and forcibly incorporated the fuzziness of the index into the category of precise mathematics. These characteristics made it difficult to deal with the randomness and uncertainty of indicators. The method in this article used the cloud model to consider the ambiguity and randomness in the HSE 
evaluation work, and the cloud diagram of the model in the article vividly and intuitively depicted these characteristics of the evaluation [47]. The calculation results are displayed in two ways: similarity and cloud graph, which is convenient for decision makers to use evaluation information.

\section{Conclusions}

To accurately evaluate the HSE risk of MSTTPs and effectively deal with their randomness and ambiguity, the SEWM and the cloud model were employed. In this paper, for the first time, an index system for MSTTPs at the construction stage was constructed from three aspects of health, safety, and environment via a literature review and expert interviews. Based on the genetic reduction algorithm of rough set theory, this paper retained the core evaluation index, effectively reduced the workload of the evaluation process, and enhanced the practicability of this method. Then, weights were calculated by the SEWM with the advantages of strong explanatory power and high accuracy of calculation results. To clarify the randomness and ambiguity in the evaluation, the HSE risk level was determined by the cloud similarity. This model was applied to the Donghu Deep Tunnel Project in Wuhan, China, and the results demonstrated that its HSE risk level was medium, which was acceptable. The index related to construction safety had the largest weight. A humid environment, improper power utilization, and sludge and mud pollution were found to be the most influential risk indicators, which should be key points in HSE risk management of MSTTPs. The risk level could be intuitively and qualitatively judged by the figure evaluation cloud, providing a vivid and rapid evaluation tool for the emergency decision-making of project managers, and the risk level could be quantitatively judged by the calculation of cloud similarity. Through the comparison with gray correlation degree, set pair analysis, and fuzzy comprehensive evaluation method evaluation results, we prove the scientificity and effectiveness of the proposed model.

The construction process of MSTTPs has obvious stages and is highly dynamic, so the HSE risk factors and risk levels also have obvious stages and are highly dynamic. The accurate description of these stages and dynamics will further improve the application and promotion value of the research results achieved in this paper. Due to the difficulty in obtaining engineering data, qualitative indicators were used in this research. Therefore, determining how to select and use quantitative indicators to describe the HSE risk of MSTTPs will be explored in future research.

\section{Data Availability}

The case analysis data used to support the findings of this study are available from the corresponding author upon request.

\section{Conflicts of Interest}

The authors declare that there are no conflicts of interest regarding the publication of this paper.

\section{Acknowledgments}

This study was supported by the National Key R\&D Program of China (2018YFC0704301) and the Science and Technology Project of Wuhan Urban and Rural Construction Bureau, China (201943).

\section{References}

[1] H. Wang, C. Mei, J. Liu, and W. Shao, "A new strategy for integrated urban water management in China: Sponge city," Science China Technological Sciences, vol. 61, no. 3, pp. 317329, 2018.

[2] G. H. Wang, Y. Chen, J. H. Zhou et al., "Discussion on application and development trend of deep tunnel drainage technology," China Water and Wastewater, vol. 32, no. 22, pp. 1-13, 2016.

[3] J. Liu, L. Xia, H. Wang, W. Shao, and X. Ding, "Typical case analysis of deep tunnel drainage system in urban area," Chinese Science Bulletin, vol. 62, no. 27, pp. 3269-3276, 2017.

[4] M. L. Huang, Z. E. Zhang, and Z. S. Tan, "Construction model of deep tunnel for urban flood control and drainage in China," China Civil Engineering Journal, vol. 50, no. 1, pp. 76-81, 2017.

[5] A. Dewan, P. Soren, R. B. N. Mathias, and O. Jacob, "Why does the offshore wind industry need standardized HSE management systems? An evidence from Denmark," Renewable Energy, vol. 136, pp. 691-700, 2019.

[6] A. Motaghifard, M. Omidvari, and A. Kaazemi, "Introducing a conceptual model for evaluating health safety environmental performance of residential buildings using the fuzzy decisionmaking approach," Environmental Monitoring and Assessment, vol. 192, no. 1, p. 19, 2020.

[7] A. Hamid, W. Saleem, G. Yaqub, and M. U. D. Ghauri, "Comparative assessment of respiratory and other occupational health effects among elementary workers," International Journal of Occupational Safety and Ergonomics, vol. 25, no. 3, pp. 394-401, 2019.

[8] P. Amir-Heidari, R. Maknoon, B. Taheri, and M. Bazyari, “A new framework for HSE performance measurement and monitoring," Safety Science, vol. 100, pp. 157-167, 2017.

[9] Q. Z. Lu and D. H. Li, "Analysis on the role of team culture construction in HSE management," Highway, vol. 63, no. 8, pp. 135-137, 2018.

[10] Z. G. Zhang and J. F. Li, "Application and demonstration of health-safety-environment risk management to underwater tunnel of Hong Kong-Zhuihai-Macao Link," Tunnel Construction, vol. 39, no. 2, pp. 189-196, 2019.

[11] W. Q. Wang, S. R. Lyu, Y. D. Zhang, and S. Q. Ma, “A risk assessment model of coalbed methane development based on the matter-element extension method," Energies, vol. 12, no. 20, pp. 3931-3960, 2019.

[12] X. Liang, W. Liang, L. Zhang, and X. Guo, "Risk assessment for long-distance gas pipelines in coal mine gobs based on structure entropy weight method and multi-step backward cloud transformation algorithm based on sampling with replacement," Journal of Cleaner Production, vol. 227, pp. 218-228, 2019.

[13] Y. R. Sun, Z. C. Dong, Y. Zhou, and S. N. Feng, "Evaluation of water resources carrying capacity in lower reaches of Yangtze River based on structural entropy method: a case study of Nanjing," Yangtze River, vol. 49, no. 7, pp. 47-51, 2018.

[14] M. J. Rezaee, S. Yousef, M. Eshkevari, M. Valipour, and M. Saberi, "Risk analysis of health, safety and environment in chemical industry integrating linguistic FMEA, fuzzy 
inference system and fuzzy DEA," Stochastic Environmental Research and Risk Assessment, vol. 40, no. 2, pp. 231-242, 2019.

[15] J. F. Zhou, S. Y. Hu, J. K. An et al., "Research on key indexes of power supply level of medium-voltage distribution network based on entropy-weighted method and grey relational degree," Bulletin of Science and Technology, vol. 35, no. 12, pp. 101-104, 2019.

[16] Q. Wu, L. Wang, N. Jin, N. Li, and X. Hu, "Social stability risk evaluation of major water conservancy projects in fragile ecoenvironment regions," Applied Ecology and Environmental Research, vol. 17, no. 4, pp. 9097-9111, 2019.

[17] X. Q. Yang and S. C. Zhang, "Manned space engineering project based on fuzzy analytic hierarchy process-particle swarm optimization research on identification of important processes," Science and Technology Management Research, vol. 39, no. 24, pp. 217-224, 2019.

[18] D. Liu, D. Wang, J. Wu et al., "A risk assessment method based on RBF artificial neural network-cloud model for urban water hazard," Journal of Intelligent \& Fuzzy Systems, vol. 27, no. 5, pp. 2409-2416, 2014.

[19] K. K. Peng, "Risk evaluation for bridge engineering based on cloud-clustering group decision method," Journal of Performance of Constructed Facilities, vol. 33, no. 1, Article ID 04018105, 2019.

[20] H.-C. Liu, L.-E. Wang, Z. Li, and Y.-P. Hu, "Improving risk evaluation in FMEA with cloud model and hierarchical TOPSIS method," IEEE Transactions on Fuzzy Systems, vol. 27, no. 1, pp. 84-95, 2019.

[21] C. L. Themann and E. A. Masterson, "Occupational noise exposure: a review of its effects, epidemiology, and impact with recommendations for reducing its burden," The Journal of the Acoustical Society of America, vol. 146, no. 5, pp. 3879-3905, 2019.

[22] E. Jonsson, B. Järvholm, and M. Andersson, "Silica dust and sarcoidosis in Swedish construction workers," Occupational Medicine, vol. 69, no. 7, pp. 482-486, 2019.

[23] P. Wolkoff, "Indoor air humidity, air quality, and health - an overview," International Journal of Hygiene and Environmental Health, vol. 221, no. 3, pp. 376-390, 2018.

[24] F. M. Fischer, C. A. Silva, R. H. Griep et al., "Working time society consensus statements: psychosocial stressors relevant to the health and wellbeing of night and shift workers," Industrial Health, vol. 57, no. 2, pp. 175-183, 2017.

[25] G. Cucchi, A. Lisardi, D. Mostacci, L. Tositti, F. Tugnoli, and S. Vichi, "Radiation protection issues in the excavation of road and railway tunnels, a preliminary assessment," Radiation Effects and Defects in Solids, vol. 171, no. 9-10, pp. 801-807, 2016.

[26] Y. S. Jiang, Z. Y. Sun, and Z. Y. Yang, "Construction risk monitoring system and parameter warning of EPB shield," Tunnel Construction, vol. 39, no. 7, pp. 1083-1089, 2019.

[27] Y. R. Yan, "Risk analysis of cutter head failure of composite EPB shield," Rock and Soil Mechanics, vol. 30, no. 8, pp. 2324-2330, 2007.

[28] G. H. Chen, W. S. Wu, S. Y. Xu, and K. Liu, “Assessment HSE risk of during sea-crossing bridges project construction based on WBS-RBS and AHP," China Safety Science Journal, vol. 23, no. 9, pp. 51-57, 2013

[29] W. Q. Wei, L. Chen, and H. B. Luo, "Geological safety evaluation of cross-river metro tunnel based on clustering analysis," Journal of Civil Engineering and Management, vol. 32, no. 1, pp. 72-76, 2015.
[30] Z. M. Ding, Q. L. Liao, and C. Y. Li, "Study on risk of the construction of earth-pressure-balanced shield in metro engineering," Construction Technology, vol. 41, no. 24, pp. 64-67, 2012.

[31] B. R. Fortunato, M. R. Hallowell, M. Behm, and K. Dewlaney, "Identification of safety risks for high-performance sustainable construction projects," Journal of Construction Engineering and Management, vol. 138, no. 4, pp. 499-508, 2012.

[32] H. Lu, M. Y. Wang, B. H. Yang, and X. L. Rong, "Study on the application of the kent index method on the risk assessment of disastrous accidents in subway engineering," Scientific World Journal, vol. 2013, Article ID 360705, 10 pages, 2013.

[33] K.-C. Hyun, S. Min, H. Choi, J. Park, and I.-M. Lee, "Risk analysis using fault-tree analysis (FTA) and analytic hierarchy process (AHP) applicable to shield TBM tunnels," Tunnelling and Underground Space Technology, vol. 49, pp. 121-129, 2015.

[34] W. Liu, T. Zhao, W. Zhou, and J. Tang, "Safety risk factors of metro tunnel construction in China: an integrated study with EFA and SEM," Safety Science, vol. 105, pp. 98-113, 2018.

[35] H. Lu, Y. H. Shi, and X. L. Rong, "Discussion on safety risk assessment of shield construction in underwater tunnel," Strategic Study of CAE, vol. 15, no. 10, pp. 91-96, 2013.

[36] W. Liu, T. S. Zhao, Y. J. Zhang, Y. K. Chen, and W. Zhou, "Analysis of risk factors and countermeasures for metro shield construction," China Safety Science Journal, vol. 27, no. 10, pp. 130-136, 2017.

[37] T. Huang, F. Shi, H. Tanikawa, J. Fei, and J. Han, "Materials demand and environmental impact of buildings construction and demolition in China based on dynamic material flow analysis," Resources, Conservation and Recycling, vol. 72, pp. 91-101, 2013.

[38] O. K. M. Ouda, H. P. Peterson, M. Rehan, Y. Sadef, J. M. Alghazo, and A. S. Nizami, "A case study of sustainable construction waste management in Saudi Arabia," Waste and Biomass Valorization, vol. 9, no. 12, pp. 2541-2555, 2017.

[39] W. S. Guo, K. S. Liang, and Y. F. You, "Study of the main risks and countermeasures for the sea-crossing shield tunnel of the Taishan nuclear power station," Modern Tunnelling Technology, vol. 52, no. 6, pp. 195-202, 2015.

[40] L. Szwarcfiter, F. E. Mendes, and E. L. La Rovere, "Enhancing the effects of the brazilian program to reduce atmospheric pollutant emissions from vehicles," Transportation Research Part D: Transport and Environment, vol. 10, no. 2, pp. 153160, 2005.

[41] F. M. Shaqour and S. E. Hasan, "Groundwater control for construction purposes: a case study from Kuwait," Environmental Geology, vol. 53, no. 8, pp. 1603-1612, 2008.

[42] K.-I. Song, G.-C. Cho, and S.-B. Chang, "Identification, remediation, and analysis of karst sinkholes in the longest railroad tunnel in South Korea," Engineering Geology, vol. 135-136, no. 15, pp. 92-105, 2012.

[43] Y. X. Lv, Y. J. Jiang, W. Hu, M. Cao, and Y. Mao, “A review of the effects of tunnel excavation on the hydrology, ecology, and environment in karst areas: current status, challenges, and perspectives," Journal of Hydrology, vol. 586, Article ID 124891, 2020.

[44] Z. Pawlak and A. Skowron, "Rough sets and Boolean reasoning," Information Sciences, vol. 177, no. 1, pp. 41-73, 2007.

[45] C. Wang, M. Shao, B. Sun, and Q. Hu, "An improved attribute reduction scheme with covering based rough sets," Applied Soft Computing, vol. 26, pp. 235-243, 2015.

[46] U. K. Chakrabarti and J. K Parikh, "Risk-based route evaluation against country-specific criteria of risk tolerability for hazmat transportation through Indian State Highways," 
Journal of Loss Prevention in the Process Industries, vol. 26, no. 4, pp. 723-736, 2017.

[47] Y. P. Li, M. Q. Liu, F. Wang, and R. G. Li, "Safety performance assessment of fabricated building project based on cloud model," China Safety Science Journal, vol. 27, no. 6, pp. 115-120, 2017. 